\title{
Nanogeofísica aplicada ao monitoramento de reservatórios de hidrocarbonetos
}

\author{
Jorge Fiori Fernandes Sobreira * (PETROBRAS), Bruno Barbosa Rodrigues (PETROBRAS), Deivid dos Santos Nascimento \\ (PETROBRAS)
}

\section{Copyright 2018, SBGf - Sociedade Brasileira de Geofísica}

Este texto foi preparado para a apresentação no VIII Simpósio Brasileiro de Geofísica, Salinópolis, 18 a 20 de setembro de 2018. Seu conteúdo foi revisado pelo Comitê Técnico do VIII SimBGf, mas não necessariamente representa a opinião da SBGf ou de seus associados. É proibida a reprodução total ou parcial deste material para propósitos comerciais sem prévia autorização da SBGt.

\section{Resumo}

Nanogeophysics is a new branch of Geophysics which involves the study of the effects of injecting nanoparticles over acoustic or electromagnetic properties of the medium (sometimes both), potentially emphasizing reservoir's changes through its production cycle, thus enabling geophysical monitoring to be performed. On a typical survey, sources and receivers can be deployed either on surface or downhole, eventually on both. Applications for hydrocarbon reservoir monitoring are envisaged and are supported by physical experiments and measurements as well as by numerical modeling, which have demonstrated their potential use.

\section{Introdução}

Nanotecnologias (NT's) compreendem as tecnologias que se valem de elementos de dimensões nanométricas (isto é, $10^{-9} \mathrm{~m}$ ), tais como as nanopartículas (NP's), cujas aplicações, já consagradas em algumas áreas, têm sido propostas também para a Indústria de Petróleo, inclusive pela mobilidade dessas NP's frente às dimensões das gargantas de poros naturais, assegurada pela sua devida funcionalização. Uma destas aplicações, emergentes, considera NP's, sob determinada concentração, carreadas por fluidos como a água de injeção, para induzir ou realçar contrastes em propriedades físicas do meio, facilitando a detecção geofísica de variações de interesse. As NP's podem assim atuar como agentes de contraste (AC's) geofísico, tanto acústico quanto eletromagnético (tabela 1). O primeiro modifica a Impedância Acústica (Ip) ou Cisalhante (Is) do meio, enquanto 0 segundo modifica propriedades eletromagnéticas efetivas do meio, tais como a Permeabilidade Magnética $(\mu)$ - AC magnético -, a Permissividade Elétrica $(\varepsilon)$ - AC dielétrico - e a Condutividade Elétrica ( $\sigma)$ ou sua recíproca, a Resistividade Elétrica - AC condutivo -.

O emprego de AC's acústicos, exemplificados pelas nanobolhas, ainda enfrenta desafios, tais como a sua reduzida estabilidade frente à pressão confinante, seu tamanho, de fato micrométrico, e a sua reduzida aplicação prática (as nanobolhas de ar atuariam no sentido de neutralizar o efeito esperado de aumento da Ip, se concentradas na água de injeção).

AC's eletromagnéticos se valem das modificações nas propriedades eletromagnéticas do meio.
NP's dielétricas empregam materiais metálicos ou semimetálicos, podendo ser citados os nanotubos de titânio (Ti) pelas suas aparentes vantagens em termos de mobilidade e estabilidade frente a outras NP's dielétricas comercialmente disponíveis.

NP's condutivas empregam materiais geralmente metálicos. Variações positivas da Condutividade Elétrica com a saturação de água (Sw), porosidade e temperatura, inclusive percentualmente maiores do que aquelas associadas às propriedades acústicas/ elásticas como a velocidade da onda compressional (Vp) ou cisalhante (Vs), sugerem potencial emprego em processos de EOR (Enhanced Oil Recovery) baseados na injeção de água ou vapor. Outra aplicação atraente dos AC's condutivos é aquela destinada a "iluminar" fraturas hidraulicas induzidas em reservatórios nãoconvencionais nas proximidades de poços de tratamento: contrastes tendem a ser induzidos pela criação de uma superfície de fratura, condutiva, em meio à rocha pouco permeável, neste caso resistiva; a injeção de água contendo NP's condutivas realçaria esse contraste.

A PETROBRAS, reconhecendo o potencial destas tecnologias para aplicações em Reservatórios, manteve relacionamento com instituições de pesquisa nacionais e internacionais dedicadas, que vêm investigando desde a efetividade e economicidade do emprego de diferentes NP's como AC's geofísicos até a sua aplicação propriamente dita, que inclui o monitoramento da frente de injeção em processos de EOR. A ideia é empregar NP's eletromagnéticas como traçadores, já que elas afetariam medidas do campo eletromagnético. O rastreamento dessas NP's a partir de campos eletromagnéticos emitidos por uma fonte controlada forneceria informações importantes, por exemplo, com relação à conectividade do reservatório, à existência de barreiras de permeabilidade e ao contato óleo-água.

\section{Metodologia/ Problema Investigado}

As NP's magnéticas, pela atenção que vêm recebendo em diferentes frentes de pesquisa, são aqui destacadas. Desenvolvimentos e investigações recentes (por exemplo, Rahmani, 2012), apontam como especialmente promissor o emprego, como AC's, de NP's magnéticas com propriedades superparamagnéticas (SP).

No Brasil já são sintetizadas NP's à base de $\mathrm{Fe}_{3} \mathrm{O}_{4}$ (magnetita), que embora ferrimagnética na natureza (o que significa que exibe magnetização espontânea - ainda que em domínios internos distintos - devido a um momento magnético resultante não-nulo), passa a apresentar comportamento paramagnético, assim menos acentuado, na escala nanométrica, devido à "competição" natural entre a coercividade (de origem magnética) e a agitação molecular (de origem térmica). Tais NP's são estáveis para o intervalo de temperaturas típico dos 
reservatórios de hidrocarbonetos, assim como em meios fluidos diversos, com a sua devida funcionalização, e podem ser especificadas de acordo com a aplicação desejada.

Devido aos consideráveis momentos magnéticos elementares, costuma-se referir a tais NP's paramagnéticas como "superparamagnéticas". Estas NP's são constituídas individualmente por um cristal (monodomínio), e em conjunto, consistem de dipolos magnéticos naturalmente orientados de maneira aleatória, que, entretanto, tendem a se alinhar na presença de um campo magnético externo (quando passam a apresentar elevadas magnetizações), que venha a ser emitido a partir de transmissores e captado através de receptores, estejam eles posicionados em poços ou em superfície. Por outro lado, a elevada densidade relativa destas NP's funcionalizadas, muito próxima à da magnetita pura (que é de aproximadamente $5,1 \mathrm{~g} / \mathrm{cm}^{3}$ ), sugere que contrastes acústicos podem também ser induzidos no meio, devido à injeção de ferrofluidos (nome dado às suspensões estáveis de NP's magnéticas em fluidos carreadores, tais como a água), o que agregaria valor adicional, pela possibilidade de também de detectarem alterações em outras propriedades geofísicas do reservatório.

\section{Resultados}

Foi investigado o efeito acústico da injeção de NP's SP no reservatório. Para tal, foram conduzidos ensaios de Física de Rochas, com substituição de fluidos em meio poroso (plugues de rocha natural, com porosidade em torno de 19 a $20 \%$, no caso o arenito Berea, conhecido pela sua homogeneidade). Propriedades elásticas como $\mathrm{Vp}$ e Vs foram determinadas sob diferentes pressões confinantes (entre 1.000 e 5.000 psi, em passos de 500 psi, com acréscimo e posterior decréscimo da pressão), em 3 diferentes situações: rocha seca (aerada), rocha saturada com água destilada e rocha saturada com ferrofluido (à base de água), com concentrações de 1, 2 e $5,4 \%$ em volume. A frequência de emissão do transdutor piezoelétrico representativo da fonte foi de $700 \mathrm{kHz}$.

Enquanto foi determinado um valor de densidade $(\rho)$ do ferrofluido, com base em ensaios de PVT, em torno de $1,07 \mathrm{~g} / \mathrm{cm}^{3}$ (assim, $7 \%$ superior à da água), o valor obtido por pesagem para as amostras de rocha resultaram em 2,13 g/cm (para a rocha seca), $2,31 \mathrm{~g} / \mathrm{cm}^{3}$ (para a rocha saturada em água destilada) e $2,34 \mathrm{~g} / \mathrm{cm}^{3}$ (para a rocha saturada em ferrofluido a concentrações entre 1 e 5,4\%). Este pequeno aumento da densidade do meio efetivo saturado pelo ferrofluido tenderia, por si só, a causar a diminuição de $\mathrm{Vp}$ (devido à relação analítica entre ambos, que é inversa), o que, no entanto, tende a ser neutralizado pelo aumento mais significativo dos módulos elásticos, a saber, a incompressibilidade (k) e o módulo de cisalhamento $\left(G_{0}\right)$, que, analiticamente, têm relação direta com $\mathrm{Vp}$. Assim, o efeito final da substituição de água destilada por ferrofluido tende a ser o de aumento de $\mathrm{Vp}$ na amostra, pelo menos a concentrações não tão pequenas, como 5,4\%.

A $\mathrm{Vp}$ medida a partir da substituição de água por ferrofluido sob as concentrações testadas variou - no caso positivamente - até um máximo de $1 \%$ (ou de cerca de $2 \%$ para Ip, figura 1), sendo tal contraste percentualmente mais significativo a menores pressões confinantes, além de apresentar comportamento crescente (embora não linearmente) com o aumento da concentração. Por outro lado, variações percentualmente mais significativas, observadas para $V s$ a partir de tal substituição, inclusive superiores a $5 \%$ (à concentração de $5,4 \%$ e a pressões em torno de 1.000 psi, figuras 2 e $3)$, sugerem contrastes não negligenciáveis em termos da Impedância Cisalhante (Is), que seriam detectáveis a partir do registro de dados sísmicos multicomponentes repetidos no tempo. Foi ainda observado (fig. 3) que, enquanto a menores concentrações (como 1 ou $2 \%$ ), observa-se a diminuição de Vs com a substituição de água por ferrofluido, em função do efeito preponderante da densidade (que é maior do que a densidade da água destilada), a partir de determinada concentração (algo entre 2 e 5,4\%), já se passa a observar o aumento de Vs, o que faria supor que uma modificação no Go efetivo do sistema rocha-fluido, passa a governar tal variação, o que, a propósito, deve impactar também Vp. Por outro lado, a simples troca de um fluido puro pelo outro (ar pela água, por exemplo) em vez de por uma suspensão como o ferrofluido, em nada alteraria, na prática, o Go efetivo da amostra.

Este comportamento próprio de Vs em função da concentração, distinto daquele observado para Vp, traz, como consequência, que parâmetros elásticos (indicadores) relacionados à diferença entre $\mathrm{Vp}$ e $\mathrm{Vs}$, como a razão de Poisson $v$ (e provavelmente também a combinações, como é o caso de $\mathrm{lp}$-Is) seriam úteis ao estudo de processos de substituição de fluidos, a qual ficaria ressaltada pela maximização destes parâmetros sob determinada concentração "ótima" do ferrofluido, certamente inferior a $5,4 \%$, de acordo com o que foi observado, lembrando que

$$
v=\frac{\left(0.5 V_{p}^{2}-V_{S}^{2}\right)}{\left(V_{p}^{2}-V_{S}^{2}\right)}
$$

As constatações acima sugerem que além do contraste eletromagnético, também um contraste acústico/ elástico (mensurável por levantamentos sísmicos, especialmente se multicomponentes) seria induzido no reservatório pela injeção do ferrofluido, ampliando assim o potencial de detecção por métodos geofísicos.

Tal detecção de variações acústicas (sísmicas) seria favorecida pelo emprego de ferrofluidos com concentrações mais elevadas (não obstante o aumento de custo correspondente), mas também pelo emprego de sistemas de monitoramento sísmico de elevada repetibilidade 4D (por exemplo, sistemas permanentes), pela aquisição de dados sísmicos multicomponentes (de superfície ou de poço), que incluíssem a obtenção de dados da onda PS além da PP, e preferencialmente em reservatórios não tão profundos a ponto de a pressão confinante acarretar menor detectabilidade do contrate induzido. 


\section{Discussão e Conclusões}

A avaliação da viabilidade técnica de aplicações práticas de NP's para reservatórios pode ser empreendida a partir de diferentes abordagens e escalas, que incluem a modelagem numérica, experimentos físicos em escala reduzida (em bancada e/ ou em laboratório, empregando análogos de rochas-reservatório), experimentos em escala intermediária e por fim, testes-piloto em escala de campo.

A realização de um teste piloto em um campo petrolífero, preferencialmente terrestre e submetido à injeção de água, enquanto fluido carreador das NP's, ajudaria a demonstrar o conceito da indução de contrastes geofísicos pela injeção de tais partículas, teste este que seria realizado numa base poço a poço ou poçosuperfície. Variáveis a serem consideradas num estudo de viabilidade técnica previamente a tal piloto, baseado em modelagem numérica e/ ou experimental, incluem a concentração ótima de NP's, necessária à sua detectabilidade, em função do contraste induzido no reservatório (relacionada ao custo), o volume e/ ou vazão do fluido carreador (relacionados a aspectos de Geoengenharia) e os valores intrínsecos da propriedade física tanto para as NP's a serem empregadas como para o reservatório a ser injetado (relacionados à efetividade do material e à detectabilidade do contraste induzido).

Uma possibilidade adicional envolve a tomografia eletromagnética aplicada às NP's, que parte do princípio que, enquanto nas baixas frequências predominam processos de difusão, nas mais altas o comportamento do campo eletromagnético pode ser aproximado pela propagação de uma onda com velocidade expressa em termos da permeabilidade magnética e da permissividade elétrica do meio. De acordo com trabalhos que investigaram este tipo de aplicação (por exemplo, AlShehri et al., 2013), a resposta de um meio poroso injetado por ferrofluido à passagem da onda eletromagnética emitida por uma fonte pulsada de alta frequência é expressa na forma de diferentes tempos de chegada, os quais permitiram identificar claramente a frente de injeção do ferrofluido: neste caso, o aumento de $\mu$ efetivo do meio, por exemplo, acarretaria retardos no tempo de trânsito da onda eletromagnética.
NT's que empregam NP's como AC's geofísicos, aplicadas a reservatórios de hidrocarbonetos, constituem tecnologias emergentes na Indústria de Petróleo com efetivo potencial de aplicação. Dentre estas, parecem especialmente promissoras as NP's magnéticas, cuja injeção, no reservatório, induz contrastes eletromagnéticos, detectáveis por levantamentos dedicados. Investigações baseadas na Física de Rochas indicaram que além destes últimos, contrastes acústicos/ elásticos são também induzidos no meio, o que estende as possibilidades de detecção, também por levantamentos de dados sísmicos, preferencialmente multicomponentes, que permitam obter mais diretamente parâmetros como Is, Ip-ls e $v$. Estes achados corroboram a importância da integração de métodos geofísicos na obtenção de resultados mais consistentes.

\section{Agradecimentos}

Agradecimentos são devidos à PETROBRAS por ter autorizado a submissão deste trabalho, e às equipes dos Laboratórios de Física de Rochas, de Petrofísica e de PVT (todos do Centro de Pesquisa da PETROBRAS CENPES) pelo suporte aos ensaios laboratoriais.

\section{Referências}

Al-Shehri, A. A., Ellis, E. S. M., Servin, J. M. F., Kosynkin, D. V., Kanj, M. Y., Schmidt, H. K., 2013. Illuminating the reservoir: magnetic nanomappers. Saudi Aramco Journal of Technology, fall 2013, 40-47.

Rahmani, A. R., 2012. Theoretical study of magnetic nanoparticles: implications for enhanced reservoir imaging (estudo feito para o Advanced Energy Consortium, confidencial). 
Tabela1 - Comparação dos principais agentes de contraste geofísicos associados a nanopartículas.

\begin{tabular}{|c|c|c|}
\hline $\begin{array}{l}\text { NANOPARTICULA/ TIPO DE } \\
\text { CONTRASTE INDUZIDO }\end{array}$ & PROPRIEDADE FÍSICA DE INTERESSE & $\begin{array}{l}\text { GRAL DE MATURIDADE TECNOLÓGICA } \\
\text { PARAAPLICAÇÃO REAL }\end{array}$ \\
\hline Acústica & Impedâncla accística (Ip) & $\begin{array}{l}\text { Mediano a balso: existem questōes alnda em } \\
\text { aberto (vide terto) }\end{array}$ \\
\hline Resistlva & Reslstlvidade (ou conduttvldade, o) elétrica & $\begin{array}{l}\text { Mediano (já são conhecidos bons resultados de } \\
\text { monitoramento deste tlpo, por excmplo no } \\
\text { campo de Dom João - BA, embora não } \\
\text { empregando XP's) }\end{array}$ \\
\hline Dielétrica & Permissividade elétrica ( $\mathrm{E}$ ) & $\begin{array}{l}\text { Mediano a baiso (mesmo agentes com forte } \\
\text { permlssividade elétrica provocam vartaçóes } \\
\text { alnda sutds no melo, necessitando-se melhorar a } \\
\text { sensibilidade das fcrramentas e métodos de } \\
\text { detecçāo) }\end{array}$ \\
\hline Magnética & Permeabilidade magnética $(\mu)$ & $\begin{array}{l}\text { Mediano (agentes de contraste magnéticos já } \\
\text { disponívels no Brasill- USP) }\end{array}$ \\
\hline
\end{tabular}

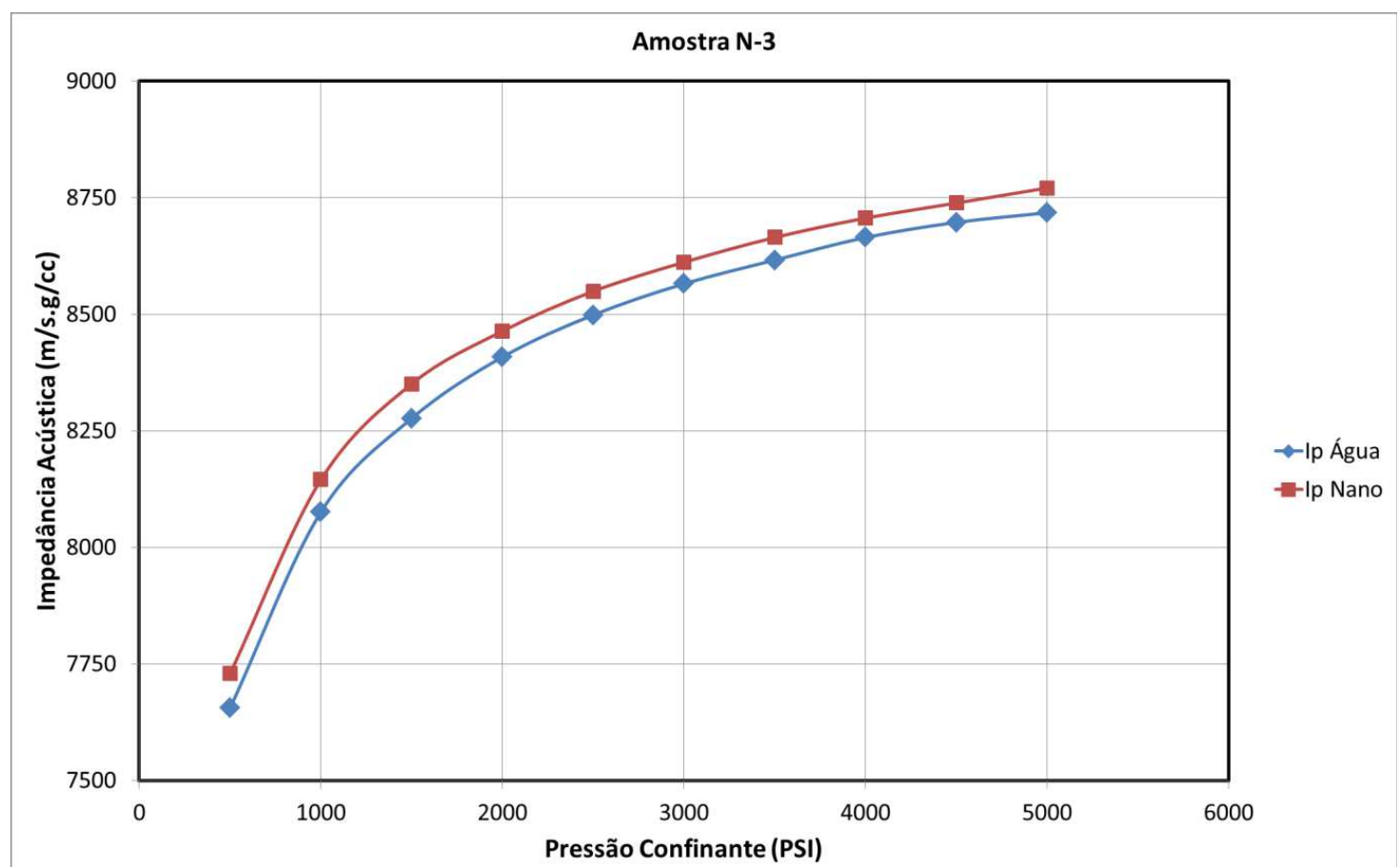

Figura 1 - Variação de Ip com a pressão confinante, derivada de ensaios petrossísmicos conduzidos pela PETROBRAS em laboratório, com a substituição de água destilada ( "Ip água") por ferrofluido à concentração de 5,4\% em volume ("Ip Nano") em amostra do arenito Berea. Os valores para a água de formação, natural, seriam ligeiramente diferentes. As diferenças entre os fluidos são percentualmente mais pronunciadas nas mais baixas pressões. 


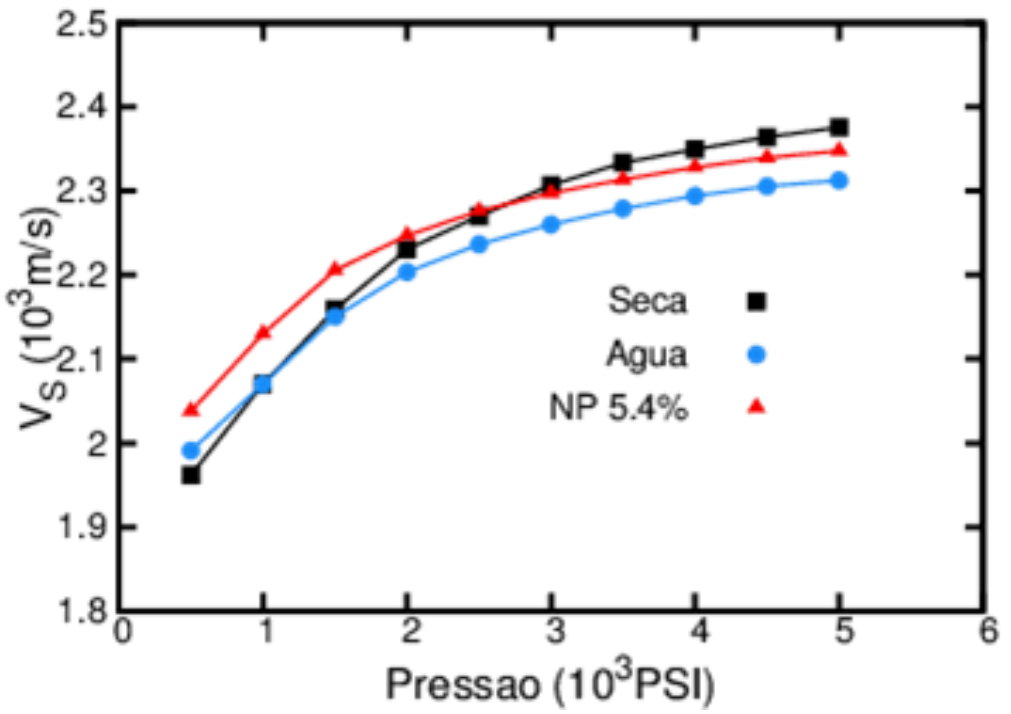

Figura 2 - Variação de Vs em função da pressão confinante, derivada de ensaios petrossísmicos conduzidos pela PETROBRAS em laboratório, com a substituição de fluidos: ar (rocha seca), água destilada ("Água") e ferrofluido à concentração de 5,4\% em volume ("NP 5,4\%") em amostra do arenito Berea. Os valores para a água de formação, natural, seriam ligeiramente diferentes. As diferenças entre os fluidos são percentualmente mais pronunciadas nas menores pressões. A obtenção de valores menores para a amostra com água em relação à amostra seca reflete tão somente a sua maior densidade global, que faz diminuir o valor de Vs, tal como analiticamente previsto (considerando $G_{0}$ da rocha praticamente inalterado pela substituição de fluido).

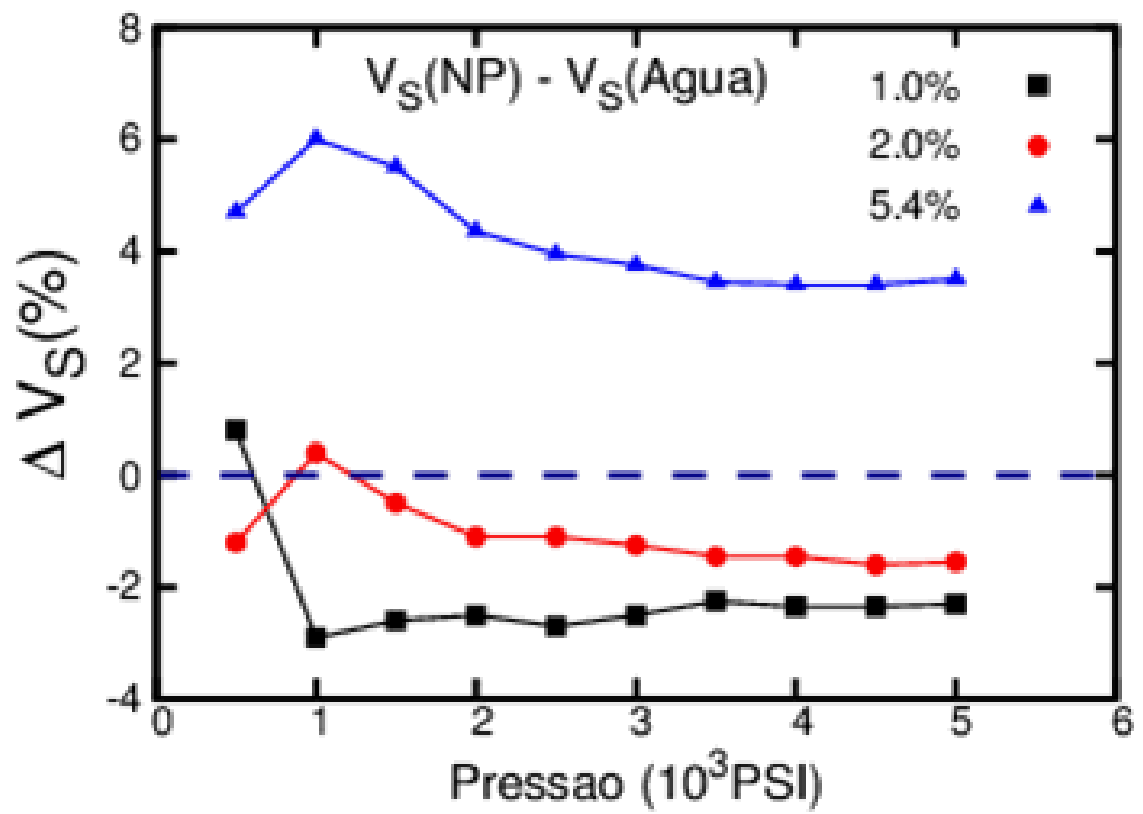

Figura 3 - Variações de Vs em função da pressão confinante, derivadas de ensaios petrossísmicos realizados pela PETROBRAS, com a substituição de água destilada por ferrofluido a diferentes concentraçòes (1, 2 e 5,4\%) em amostras do arenito Berea, onde se observa uma mudança no comportamento geral entre as concentrações mais elevadas $(5,4 \%)$ e as menos elevadas (1 e $2 \%)$. Maiores variações são observadas em geral a menores pressões confinantes. 